\title{
Theme: An Approach for Aspect-Oriented Analysis and Design
}

\author{
Elisa Baniassad and Siobhán Clarke \\ Department of Computer Science \\ Trinity College, Dublin 2, Ireland \\ \{Elisa.Baniassad, Siobhan.Clarke\}@ @ cs.tcd.ie
}

\begin{abstract}
Aspects are behaviours that are tangled and scattered across a system. In requirements documentation, aspects manifest themselves as descriptions of behaviours that are intertwined, and woven throughout. Some aspects may be obvious, as specifications of typical crosscutting behaviour. Others may be more subtle, making them hard to identify. In either case, it is difficult to analyse requirements to locate all points in the system where aspects should be applied. These issues lead to problems achieving traceability of aspects throughout the development lifecycle. To identify aspects early in the software lifecycle, and establish sufficient traceability, developers need support for aspect identification and analysis in requirements documentation. To address this, we have devised the Theme approach for viewing the relationships between behaviours in a requirements document, identifying and isolating aspects in the requirements, and modelling those aspects using a design language. This paper describes the approach, and illustrates it with a case study and analysis.
\end{abstract}

\section{Introduction}

The intent of aspect-orientation is to allow developers to encapsulate system behaviour that does not fit cleanly into the particular programming model in use; it is aimed at breaking the hegemony of the dominant decomposition.

Behaviour that cannot be encapsulated because of its impact across the whole system is called crosscutting behaviour. Before encapsulating crosscutting behaviour into an aspect, the developer must first identify it in the requirements. This is difficult, because, by their nature, aspects are tangled with other behaviours, and are likely to be described in multiple parts of the requirements document. Using intuition or even domain knowledge is not necessarily sufficient for identifying the potentially broad range of aspects within a reasonable amount of time. For example, it required significant effort to identify and characterise that prefetching could be modeled as an aspect in the FreeBSD operating system [7].

When identifying aspects early in the lifecycle, developers can currently apply three approaches. The common approach for aspect-identification is to look for the objects in a system first, and then attempt to spot the tangled and scattered behaviour as it becomes evident. This is an adhoc approach that is likely to necessitate re-design as aspects are discovered late in the design process. Alternatively, before the design process starts, developers might scan requirements for mentions of typical aspect-style behaviour, such as logging, tracing, or debugging functionality. This only covers a narrow range of potential aspects; it does not help with identifying crosscutting behaviour that does not fall into these categories, or that might be domain specific. To address this need, a developer might start by applying an aspect-oriented requirements engineering technology, and target non-functional requirements as an initial set of aspects $[13,11,16]$. However, there are likely to be many functional requirements in the system that probably break down into complicated and interrelated behaviours. Would any of those be aspects? Where would they crosscut the system?

We believe that in order to identify and model a broad range of aspects early in the lifecycle, and assess where they crosscut the system, developers need support for analysis of the relationships between all behaviours described in requirements documentation. They also need support for translation of the results of the analysis into design models which can then be implemented in code.

The model we propose is the Theme approach. Theme provides support for aspect-oriented development at two levels. At the requirements level, Theme/Doc provides views of requirements specification text, exposing the relationship between behaviours in a system. At the design level, Theme/UML [4, 6] allows a developer to model features and aspects of a system, and specify how they should be combined. Our central claim is that Theme/Doc allows the developer to refine views of the requirements in order to reveal which functionality in the system is crosscutting, 


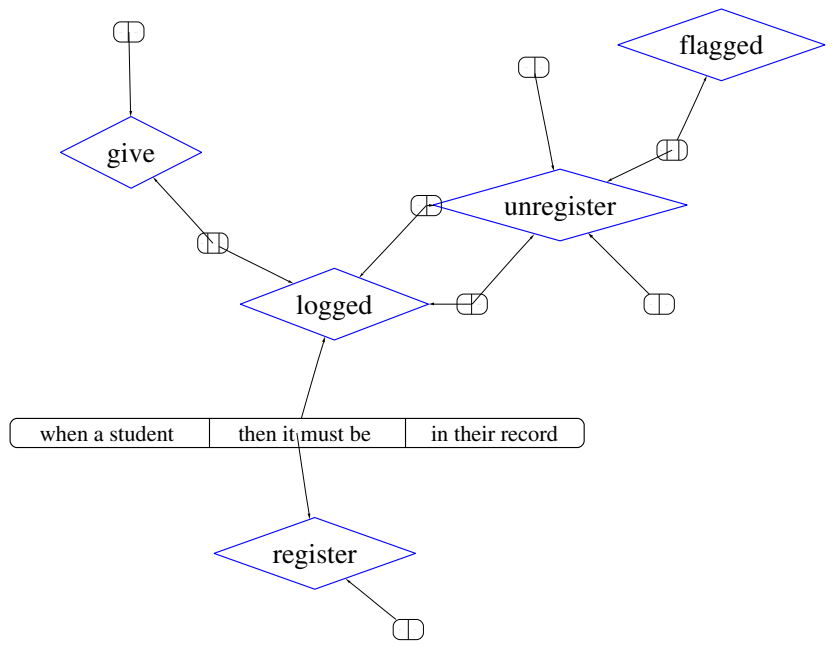

Figure 1. Action View of CMS Requirements

and where in the system it crosscuts. We also claim that the Theme approach assists with maintaining traceability from requirements to design, since requirements map directly to Theme/Doc views which map directly to Theme/UML models. This traceability also provides cues about requirements coverage in the design.

In this paper we present the approach by means of a small example (Section 2), and provide a case study to assess its effectiveness at aspect identification, the provision of traceability and coverage cues, and scalability of the approach (Section 3). We then discuss other issues and make observations about the approach (Section 4), review related work (Section 5), and conclude (Section 6).

\section{Theme}

In the Theme approach, a theme is an element of design: a collection of structures and behaviours that represent one feature. Multiple themes can be combined or integrated to form a system [17]. Themes were introduced at the designlevel in [4]. The Theme model has two kinds of themes: base themes, which may share some structure and behaviour with other base themes, while modelling these from their own perspective, and crosscutting themes which have behaviour that overlays the functionality of the base themes. Crosscutting themes are aspects [6].

The Theme approach is divided into two segments: Theme/Doc and Theme/UML. These both operate on and refer to the same themes, but depict them at different phases of the lifecycle. Theme/Doc provides views ${ }^{1}$ and functional support for identification and depiction at the analysis

\footnotetext{
${ }^{1}$ All Theme/Doc views are generated automatically in dot [12] format given the text of the requirements document, a list of key entities, and a
}

phase, whereas Theme/UML allows standard UML modeling of relevant structure and behaviour for each theme at the design phase. ${ }^{2}$

In this section we work through a small example to illustrate the basic points of how to use Theme/Doc and Theme/UML to support the identification, design, and design checking of aspects in a set of requirements.

\subsection{Course Management System}

The Course Management System (CMS) is a very small system, with nine requirements:

- R1. Students can register for courses.

- R2. Students can unregister for courses.

- R3. When a student registers then it must be logged in their record.

- R4. When a student unregisters it must also be logged.

- R5. Professors can unregister students.

- R6. When a professor unregisters a student it must be logged.

- R7 When a professor unregisters a student it must be flagged as special.

- R8. Professors can give marks for courses.

- R9. When a professor gives a mark this must be logged in the record.

\subsection{Identifying Aspects Using Action Views}

Using the Theme/Doc tool, a developer can view the relationship between behaviours described in requirements documentation, and determine which behaviours are base, and which are crosscutting.

To help identify crosscutting behaviours we use the action view of the requirements document. Two inputs are needed to create the action view: a list of key actions identified by the developer by looking at the requirements document and picking out sensible verbs, and the requirements as written in the original document. Theme/Doc then performs lexical analysis of the text and generates the action view. ${ }^{3}$

Each action is potentially a theme to be designed separately in Theme/UML. For the CMS, we have identified five actions: register, unregister, logged, flagged, and give. Figure 1 shows the action view created by the Theme/Doc tool for the CMS. In the view we see the five actions as diamonds. The requirements from the text are shown as rounded boxes (sentence records) which contain

list of key actions. The dotty graph visualization package is used for the layout and display of the views.

${ }^{2}$ Theme/UML designs are created by a developer using an appropriate UML editor. In Theme/UML all classes and behaviour related to a particular theme are placed together in one package-style structure. These are then integrated with other themes using composition semantics such as merge and override. This is described in more detail in [4].

${ }^{3}$ Due to the lexical nature of the tool, any block of text could be defined as one requirement. With use-cases, for instance, the supporting text could be blocked as one or more requirements. 


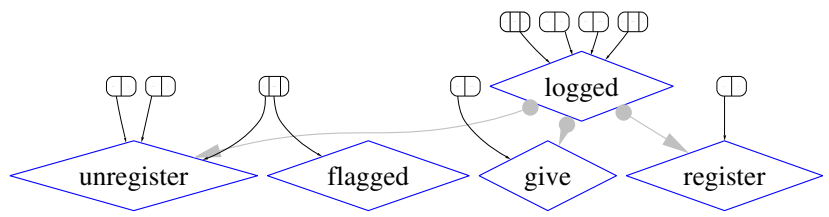

Figure 2. Clipped Action View

the words from the original sentence. If the requirement sentence contains a key action, it will be linked to the sentence record. The intent of the action view is to highlight relationships between actions; the text of the individual requirements themselves is not the point at this stage, and so is not intended to be legible. Of course, a user may choose to enlarge any requirement for their information. In this view we have chosen to enlarge R3, which reads "when a student registers then it must be logged in their record".

As we might expect, none of the actions in the requirements is isolated from the rest. They all relate in some way to the rest of the actions. The fact that they are linked shows us that there is crosscutting and tangling of behaviours within the requirements. For instance, we can see from this figure that the logged action is mentioned in four requirements, and that the register action is mentioned in two requirements.

Requirements often refer to more than one action. For instance, $\mathrm{R} 3$ refers to both the register and logged actions. We identify aspects by examining these shared requirements. Our aim is to separate and isolate groups of actions and requirements in the action view, arriving at two kinds of action/requirement groupings. The first are self-contained and have no requirements that refer to action in other groups, which we determine to be "base". The second kind are crosscutting and have requirements referring to actions in other groups.

We use the clipping functionality of the tool to achieve this separation and isolation. First, we examine each shared requirement to see which action it is more appropriately coupled with. If the requirement is too ambiguous to associate with one action or the other, we must resolve the ambiguity, either by re-writing, splitting, or refining the requirement. The requirement that links logged to register is R3, which describes logged behaviour that is added to registration behaviour. We intuit that logging is the primary behaviour of this requirement, and hence that R3 should be coupled with the logged theme. In deciding this, we have determined that logging is crosscutting and register is base. Second, we clip the arrow from R3 to register so that it is only linked to logging. That arrow is replaced by a grey arrow with a dot at its head which points from logged to register, indicating that logged crosscuts register.

We then visit each requirement that logged shares with other actions, to determine whether they belong with logged or the other action. Since we have determined that logged crosscuts register it is likely that it also crosscuts the other actions with which it is linked. We continue to snip the links between the shared requirements and the base actions, and leave them with the logged action. Finally, we arrive at our goal as shown in the clipped action view displayed in Figure 2: four actions with requirements that re-

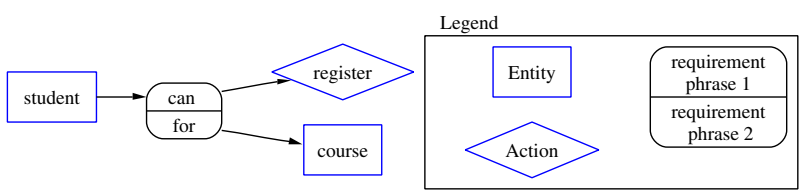

Figure 3. Theme/Doc Theme view: register

fer only to themselves and are therefore base, and one action that mentions others and is therefore crosscutting. In clipped action views, crosscutting themes are placed above the themes that they crosscut. The grey arrows indicating crosscutting in the clipped view (Figure 2) will help guide the configuration of aspect-base relationships at design.

We now make one final observation from the action groupings. In this view we see that the flagged action is linked to the unregister action. We examine the requirement they share and make the decision that the flagging functionality should be included in the professor's behaviour in the unregister theme. An alternative might have been to consider that flagging behaviour crosscut unregistering behaviour. Now, each grouping becomes a theme we wish to model using Theme/UML.

\subsection{Planning for Design Using the Theme View}

Theme/Doc's theme view is used to plan the design and modelling of the themes identified in the previous step. Theme views differ from action views in that they do not only show requirements and actions, but also show key elements of the system that will need to be considered for each theme design in Theme/UML. To construct a theme view, a developer must supply an additional set of keywords: key entities. Like the action view, the theme view is created through lexical analysis of the text of the requirements document.

Figure 3 shows the theme view of the register theme clipped of crosscutting behaviour. We can see that it has only one requirement, which mentions nothing other than registration behaviour. When reading sentences in Theme/Doc, first read the element that points into the sentence record, then read the first phrase in the record, and then read the element pointed to by that phrase. If the phrase is empty, it just means there was only a space between the first and second element, with no connecting phrase. Then read the subsequent connecting phrase, and then the element to which it points. Continue back and forth between the record and its attached elements until the record ends. To read a sentence, do not read more than one element out from the sentence record. This sentence reads "Student(s) can Register for Course(s)".

We can use the theme view to plan which classes and methods appear in our Theme/UML for register. When modelling using Theme/UML good object-oriented design practices should be used to help determine which classes and methods are described. As is evident in Figure 4, in this case, we simply decide that each action in the theme view is a method, and each entity is a class. We also make some additional design decisions to make course registration work. 


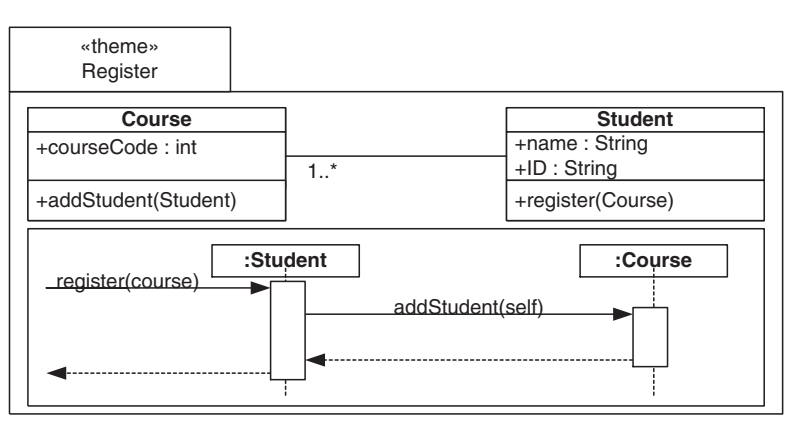

Figure 4. Theme/UML: register

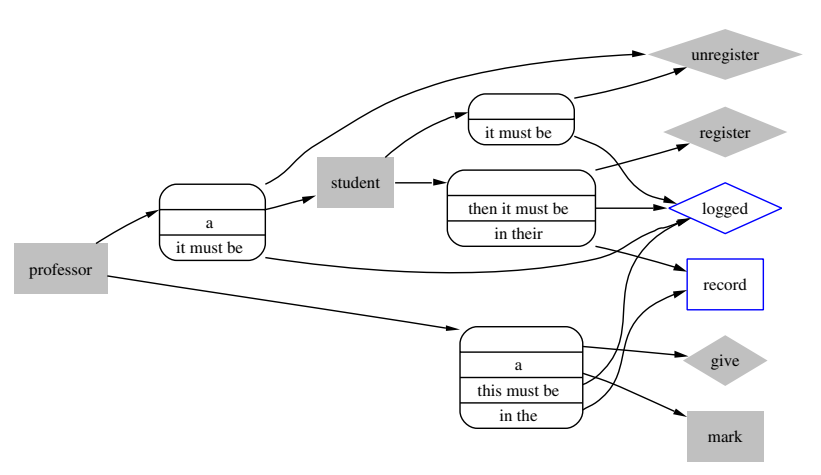

Figure 5. Theme/Doc Theme View: logged

When modelling a crosscutting theme we want to model the crosscutting behaviour in an abstract and potentially re-usable way. We do not want explicit references to any base actions or entities. The theme view for crosscutting themes helps with identification of such elements by greying out actions and entities found in other themes. The remaining white actions and entities can then be used to guide the design of the abstract crosscutting behaviour. The grey actions are also used to determine the joining, or binding of the crosscutting behaviour to the greyed-out base.

An example of this is shown in Figure 5. We can see that only elements that are unique to the logged theme are logged and record. We can now model the abstract behaviour for logging records without refering directly to registration, unregistration, students, professors or giving marks. The model for the logged theme is shown in Figure 6 in which we can see that the record element of the logged theme has become a database in this design, and that the logged action has been loosely translated into the logRecord method. Theme/UML allows reasoning about elements from a base by using templates that will be bound to real base elements at a later stage.

To determine how the crosscutting theme should hook into the base features, we look back at the theme view for logged (Figure 5). All of the grey actions are behaviours that are crosscut by the logged behaviour. The Theme/UML for logged provides the _log ( ) template method as the handle method for the base be-

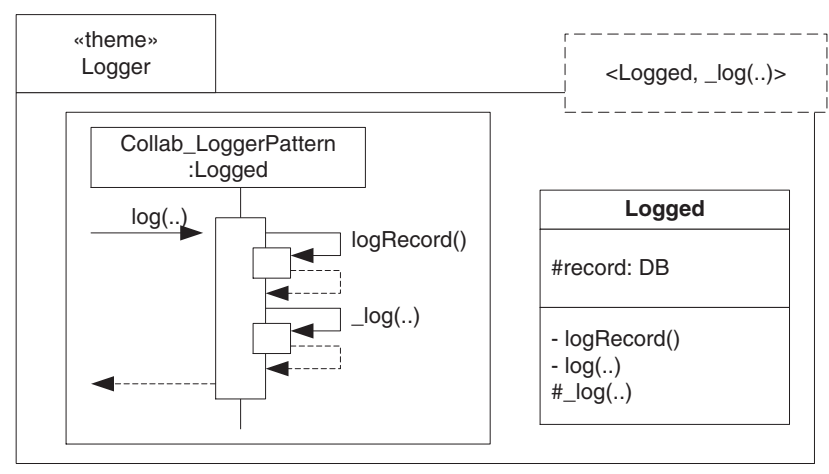

Figure 6. Theme/UML: logged

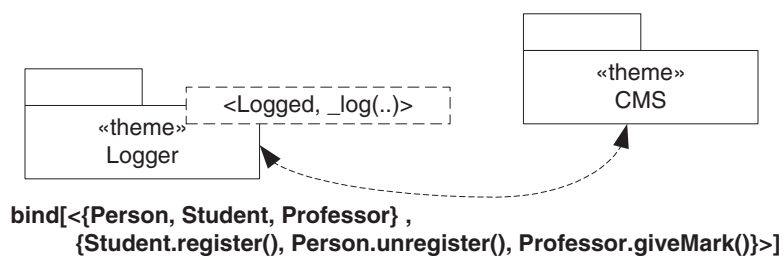

Figure 7. Composition of logged and other themes

haviour. To resolve which method the _log ( ) method actually is, we use the bind feature of Theme/UML to bind it to a concrete method from another feature of the system. So, we bind the $\_$log method to the grey actions in the logged theme view: register, unregister, and give(mark). To determine the classes to which methods belong, we can look at Figure 5 and the relevant Theme/UML models for each action. We see that the register method is associated with the Student class (also illustrated in Figure 4) and the giveMark method belongs to the Professor class. Both the Student and Professor classes are linked to the unregister method, so we use their parent class, Person, specified in a Theme/UML model not shown here, for the binding. Figure 7 depicts the bind statement to integrate the Logger theme into the $C M S$ theme, which is a product of a previous merging of all other base themes.

\subsection{Re-checking Themes Using the Augmented Theme View}

After the themes have been designed using Theme/UML, we revisit the Theme/Doc theme views for help in verifying that the design choices we made align with the requirements. To do this, we augment the Theme/Doc theme view with representations of those design decisions. To augment the register theme view, for instance, we need to add one method, and three associations. The result is shown in Figure 8. In the augmented view, "has" relationships are shown using an inverted arrow at the container element, pointing to the contained element. The "calls" relationship 


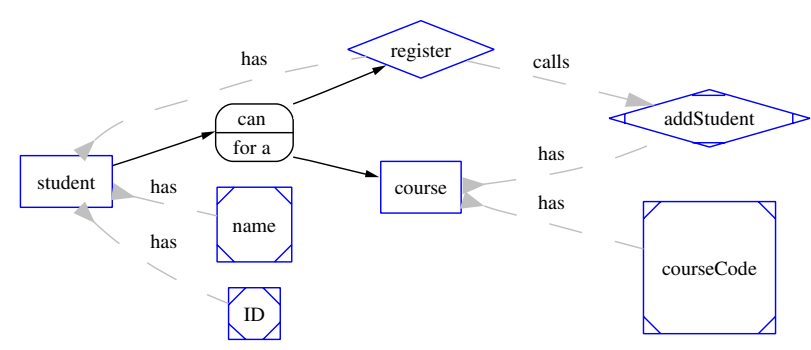

Figure 8. Augmented View: register

is shown with a dashed arrow. We've added a method to the view, which is shown as an action, and also a class, which is shown as an entity. To distinguish these as augmentations, the corners are marked. These augmentations are specified by the user and fed into the Theme/Doc tool which generates the augmented graph.

By looking at Figure 8, we can see that the Theme/UML design seems to align with the requirement shown in Theme/Doc. If there were other design elements (other classes or methods) included in the Theme/UML design, we would see them here, and would be able to assess their correctness with a view to the requirements. This view is not intended to provide any formal basis for analysis; there will not necessarily be a 1-1 mapping between requirements-level actions/entities and design-level operations/structures. Rather, it is intended to place, in the context of the requirements, the design decisions that were made. This context then gives the developer a view for verifying the design decisions themselves.

Since the Theme/UML for a crosscutting theme only includes information about the abstract crosscutting behaviour, only the structures related to the behaviour are added to the theme view. This view is shown in Figure 9, in which all augmented elements are enlarged. As before, all elements not unique to the crosscutting behaviour are shown in grey. This view has two relationships the register augmented view did not: "is-a" relationships, shown with a bi-directional arrow, and "bound" relationships which are shown with bi-directional dashed arrows, as they are in the Theme/UML bind notation. Elements that are involved in the binding but that are not shown in the view are also added. For instance, the action giveMark is in grey because it is not in the Theme/UML for the logged theme, but is included because the giveMark method is bound to the logRecord method. To assess the completeness of the design decisions for this theme we look at whether all the greyed actions are bound to the action representing the crosscutting behaviour. We also scan to see that all the structures mentioned are dealt with in some way. We can see that mark, for instance, is included in the crosscutting behaviour because it is used by the giveMark method which is bound to logRecord.

\section{Case Study}

The goals of this case study were to test the Theme approach on a larger example, and perform preliminary assessment of it in terms of effectiveness for finding aspects, support for assessment

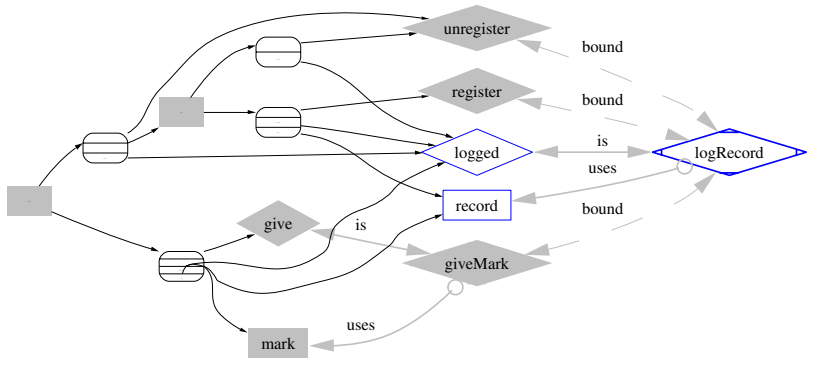

Figure 9. Augmented View: logged

of requirements coverage, facilitation of requirements traceability, and scalability. We will first give a general description of the location aware game that was the basis for the case study, and then provide results and analysis.

\subsection{Location Aware Game}

The set of requirements used in this case study are those for a location aware game called the Crystal Game, which was developed in an independent research project. The game has 89 requirements, so is roughly ten times larger than the example provided in Section 2. The game involves any number of human players, each of whom is provided hand-held devices. The game can be set in any location, as long as the location has the necessary infrastructure. The object of the game is to collect crystals that are deposited throughout the location. As a player moves around the game space, their hand-held device will alert them when they have encountered a crystal. Computer-generated characters also take part in the game. When a player encounters one of them, they will interact, and perhaps duel. When a player encounters another player, they will duel, and the loser of the duel will turn all of their crystals over to the winner. The game ends after a specified time period. The winner is decided by how many crystals each player has. There are other constraints and requirements in this game which will be of interest and will be described in later sections.

\subsection{Results}

In this section we review the steps we took to apply the Theme approach to the Crystal Game requirements.

3.2.1. Finding Themes We identified 59 actions in the game requirements, and generated an action view to examine their relationships. Based on intuition and some cursory analysis of the view, we determined that all of these actions should not be modeled as separate themes. Instead, we examined the view to determine the relationships between the actions, to decide how to group the actions into larger themes. This was a mainly analytical process, but it was supported by the action view. Because actions that share requirements are displayed close to one another in the view, we were able to examine closely located actions to assess whether they should be grouped into a common theme. 


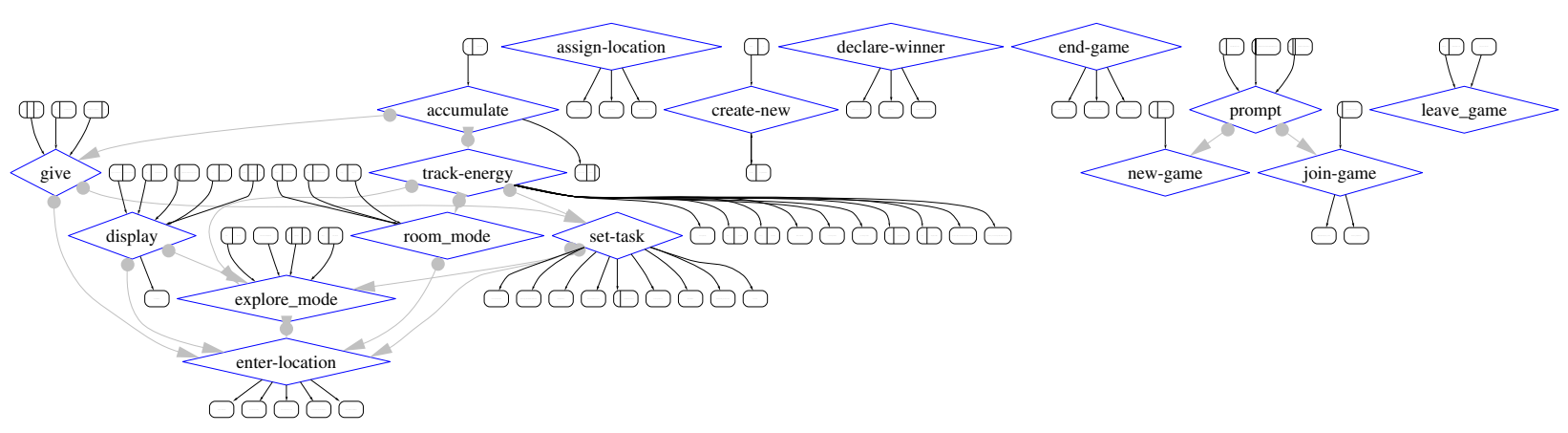

Figure 11. Clipped Action View of Major Game Actions

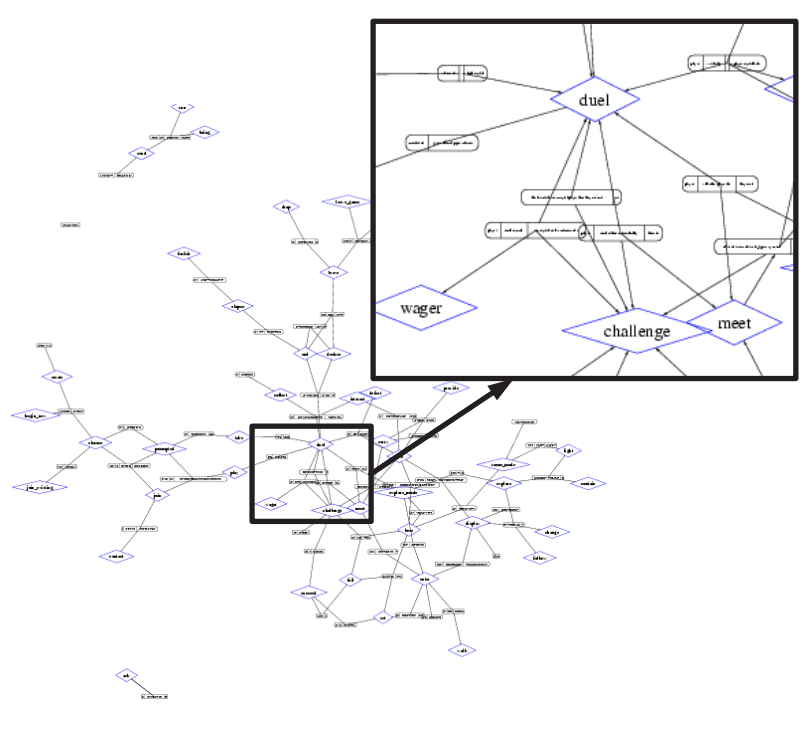

Figure 10. Game Action View: All Actions

We used the view shown in Figure 10 to perform such an assessment. This figure shows the initial action view for the game, with the centre portion of the view enlarged. The enlarged view shows four actions, duel, wager, challenge and meet. We examined the requirements they shared, considered the meaning of the actions, and determined that duel, wager, and challenge should all be grouped under the general heading of $d u e l$, since players challenge one another to duel, and wager crystals on the outcome of a duel. In that case, we classified duel as being more major than $w a-$ ger and challenge, which we saw as sub-actions of duel. The meet action was connected to duel because when players encounter one another they duel. We examined requirements shared by meeting and duelling and determined that since they were not synonymous, they should not be grouped into one theme. Later, we determined that duel and its sub-actions should be grouped under the more major action, set-task. In the end, we arrived at the view shown in Figure 11, which displays the 16 major actions which became our themes. Of those actions, five are independent, while others share requirements, and hence crosscut one another in some way.

The clipping functionality of the tool helped us investigate the major action view to determine which themes are crosscutting and which are base. In the case of the prompt theme this was straightforward. The prompt theme (shown to the right of Figure 11) shared requirements with two other themes, new-game and joingame. By examining the shared requirements it could be seen that the prompting behaviour crosscut these two themes.

As is visible on the left side of Figure 11, there are several related themes. To determine which of those was crosscutting, we began by assessing the requirements between the explore-mode and enter-location themes. We determined that explore-mode crosscut enter-location. By continuing to examine themes that shared requirements with enter-location we further determined that room-mode was crosscutting, as was give, accumulate, settask, and display. We then examined the remaining shared requirements, and encountered themes that crosscut other crosscutting themes. For instance, the track-energy theme was determined to crosscut set-task, room-mode and explore-mode, all of which crosscut enter-location. There are five themes that crosscut other crosscutting themes: display, set-task, give, track-energy and accumulate. $^{4}$

3.2.2. Modelling and Composing Themes The theme view was used to drive the modelling and composition semantics for design of the game using Theme/UML. Figure 12 shows the Theme/UML for the give theme. The give theme handles the situation in which a player or a character gives a crystal to another player. This happens in three situations: when a player meets a character, the character gives them a crystal; when a player loses to another player, the loser gives the winner a crystal; and when a player completes a task for a character, the character gives the player a crystal.

To ensure that the developer carefully considers the order in which crosscutting themes are composed with base themes, Theme/UML allows only one crosscutting theme per composition. We therefore needed to inspect the crosscutting relationships to determine the order of binding. For this we used the clipped action view shown in Figure 11. In this view, the themes are positioned hierarchically, based on whether they crosscut one another. The grey arrows indicate which themes crosscut other themes. We can see, for instance, that there are no grey arrows extending from

\footnotetext{
${ }^{4}$ AspectJ [1], allows specification of join-points in aspect code as well as core code, allowing for the expression of aspects that weave into other aspects. This can be done by providing the aspect name and method in the pointcut statement of the aspect to be crosscut.
} 
enter-location, which indicates that it is base functionality. This is also suggested by the fact that enter-location is positioned lowest in the graph. To determine the binding order, we begin with the lowest themes, and work to the highest, incrementally binding in one crosscutting theme at a time. To determine what is first in the binding we identify the themes that crosscut only that theme (room-mode and explore-mode), and placed those first, and second in the binding order. The final bindings were done with the "more" crosscutting themes: display, give and track-energy. The very last binding is of accumulate, since it crosscuts the give and track-energy themes.

The give theme bindings are also shown in Figure 12. Give is bound fifth in the general order, and is bound to an already composed theme, enterLocation-exploreMode-roomModedisplay-setTask. As the name suggests, the composed theme is the product of binding enter-location to its closest four crosscutting themes.

3.2.3. Checking Theme/UML Finally, we used the augmented theme view to check the validity of the design choices we made. An example of this is shown in Figure 13. In this figure we can see that four elements have been added to the view: giver, receiver, receive, and item. The relationships from the Theme/UML are also shown. We can see that all the grey actions have been bound to give, except for wager and duel, which are drawn in through lose. We can see that both players and characters are givers, but only a player is bound to receiver.

\subsection{Analysis}

In this section we discuss how the results of the application of the Theme approach reflect on the four areas of interest: effectiveness at support for aspect identification, requirements coverage, traceability, and scalability.

\subsubsection{Effectiveness of Support for Aspect Identification} Through the application of the Theme approach, we were able to identify eight aspects: explore-mode, room-mode, accumulate, track-energy, give, set-task, display and prompt. Had we carefully read the requirements document we may have identified seven of these behaviours as aspect behaviours since they provide tracking or logging style functionality. However, it is unlikely that we would have identified the give functionality as an aspect because mentions of the give action are spread throughout the document, and it might have been difficult to recall that the same abstract behaviour is occurring with relation to different system features. Also, since in the document text it is described as a consequence of other actions, such as meeting, and duelling, it is possible that we would have automatically thought of give as a method in those actions. It wouldn't have been until we were modelling or implementing it that we would have noticed its crosscutting nature. We can see from Figure 12 that the give functionality works as an aspect, as it can be expressed in an abstract way, and can be overlayed on the appropriate behaviour through bindings.

We also found our approach effective support for determining the binding order for multiple crosscutting themes. This may be otherwise difficult to determine.

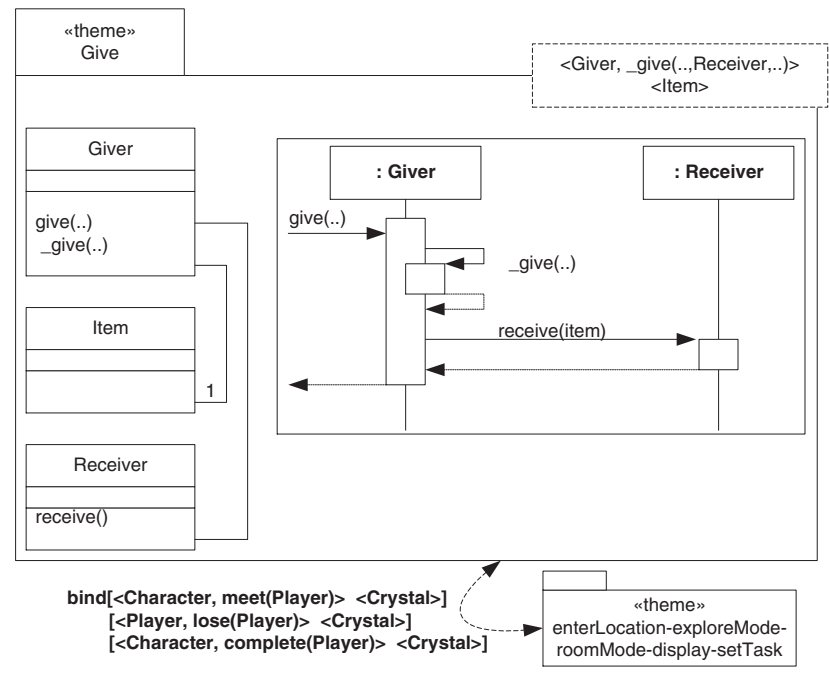

Figure 12. Theme/UML: give

3.3.2. Requirements Coverage We were initially concerned that it may also be difficult to assess whether all the requirements have been associated with a theme. We noted that the action view can be used to monitor requirements coverage, because if a requirement is not associated with a theme it is orphaned in the view. By orphaned, we mean that only the sentence record for the requirement appears, without being linked to a diamond-shaped action. We found that a requirement could be orphaned in two ways. Orphans can appear in the initial action view if the requirement contains no key actions. This may happen if it refers to another action, but does not mention it explicitly. By inspecting the requirement we can identify the requirement's original location in the text, and can read the requirement in context to determine to which action it refers. The other way orphans can appear is when forming the major action view. As major actions are identified, they are added to a new list of keywords. The minor actions that have been grouped under the major action will be annotated so that they will be linked to the major action in the view. Minor actions that are not grouped with major actions will disapear, and their requirements will appear to be orphaned. We systematically visited the orphaned requirements to determine whether any of their minor actions should be promoted to major, or whether to group those requirements under other major actions.

3.3.3. Traceability We found that the Theme approach provides traceability by explicitly linking portions of a requirements document to their outcome in a design model. Action views directly represent the requirements documentation, and are traceably refined into major action views. These maintain both the original content of the requirements, and also a developer's choices about how the requirement should be grouped into features. Major action views can then be transformed into theme views, which have a 1-to-1 mapping to their modelling in Theme/UML. As a final step, the design choices made at the modelling level are then added into the theme view to form the augmented view, which places those design choices in context with the requirements. The developer 


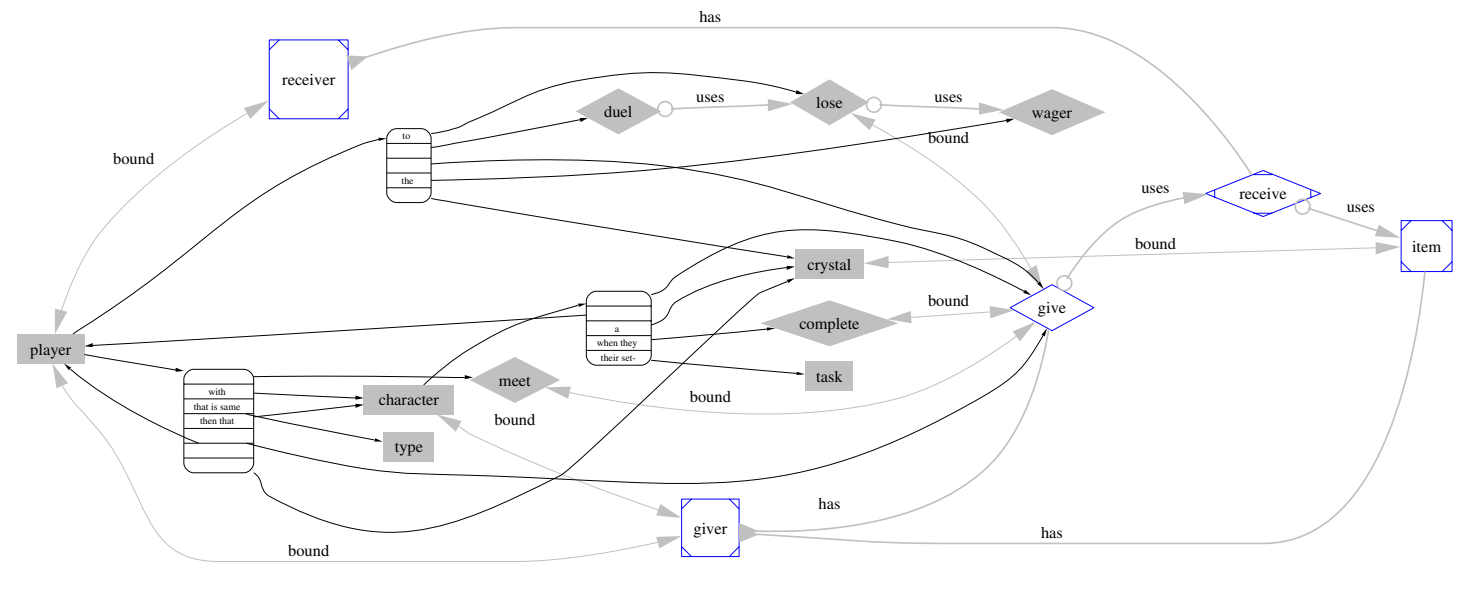

Figure 13. Augmented View: give

can examine any Theme artifact and view backward and forward traceability links. For instance, when looking at a theme view we obtain backwards mappings by inspecting the sentences displayed to find their location in the original text, and by observing which actions were deemed to be minor. We can also obtain forward mappings identifying the Theme/UML that models the view based on their common name.

For example, in Figure 13 we see the augmented view for the give theme. If we inspect the sentences shown in this view we would find that they are sentences 4, 18 and 69 from the original text. We can see that actions duel, lose, wager, meet, and complete are all contained in other themes. By visiting the action view we can infer that they were deemed minor actions since there is no major theme named after any of them, and that three were grouped under the set-task theme, and one was grouped under the enter-location theme. This is further confirmed by viewing the give Theme/UML shown in Figure 12.

3.3.4. Scalability of Action Views In the small CMS example, almost every action became a theme. This allowed for easy association of requirements with themes, and for easy viewing. However, in the larger game example, we classified actions into two types: major and minor. Major actions became themes, while minor actions were slotted to become methods within a theme. When forming the action views for this larger example, we chose only to use the major actions to form the view, rather than viewing all the major and minor actions. This allowed us to determine and assess the relationships between themes without the added "noise" of the minor actions.

Were we to scale the requirements further, it would be necessary to apply other approaches, since it would not be feasible to fit an entire action view for a very large system onto a screen or a page. In this case, query functionality, provided by the Theme/Doc tool, is needed to form sub-views that could be examined separately from the entire action view.

The current approach has essentially provided two "zoomlevels" of action view; a developer can zoom-in to see all the actions, or can zoom-out and just see the major actions. It would likely be useful, in a larger system, to provide more degrees of zooming so at some level the entirety of the system could be seen in one view.

\section{Discussion}

In this section, we provide further discussion of issues we noted while evaluating the Theme approach.

\subsection{Synonyms}

Synonyms are handled through a synonym dictionary which, for the sake of the action view, automatically augments the requirements text so that the correct associations will be made. This is more complicated when two words are the same but have different meanings in terms of the system. For instance, the term give was used in the Crystal Game not only for giving crystals, but also for giving audio and visual signals to players. The action view helped identify instances where this occured, because the common action brought together other actions which, upon analysis, should not be linked. For instance, the common term give brought closer together accumulate and prompt. We could intuit from having read the requirements document that these two actions should be unrelated. When inspecting the relationships around the give action, it was clear that the term was being used in different senses. We then used the annotation feature of the tool to replace the audio sense with the term give-audio. These annotations are not shown in the theme view.

\subsection{Scattered Requirements}

When analyzing the requirements for the context aware game, we found that not only did behaviours crosscut other behaviours (such as give crossutting the meet action in the enter-location theme), but behaviours were also described in a way that crosscut the requirements. The give behaviour was described in three locations in the text: at lines 4,18 , and 69 . This is because the original authors of the requirements did not see the give behaviour as a major enough behaviour to discuss separately, though they did orga- 
nize the document in terms of the features as they saw them. This scattering was also true of the other crosscutting themes, with the exception of room-mode and explore-mode which were described in their own sections.

\subsection{Ambiguities Found in Requirements}

We found that the Theme approach helped us identify ambiguities in the original requirements specification. While refining the 59 actions into the 16 themes we found that there were subtle ambiguities in the initial requirements document. For instance, we found that it was not explicitly mentioned how crystals were collected by a player, unless the crystal was actually given to them by another player or a game character. One requirement mentioned picking up crystals ("a player explores the world and picks up crystals"), and another mentioned the accumulation of crystals ("a player collects crystals by discovery in a location, or when a player or character gives one to them.") Though it was implied, there was no specific description of a player actually picking up a crystal when they discover it in a location. This subtle ambiguity was discovered when we saw that the pick-up action, and the collect action were not located close to one another in the view shown in Figure 10, though we knew intuitively that they should be related. This was highlighted because the collect action was closely placed to the give action.

\subsection{Evolution of Requirements}

There are two situations in which requirements can change: during the requirements gathering and analysis stage, or after modeling has begun. In either situation, the evolution can be handled by re-generating the views for the set of requirements, and deciding under which themes the new requirements should fall. A developer would follow the same process as outlined for the original requirements set for the new or changed set of requirements.

\section{Related Work}

The intent of this work is to support analysis of requirements documents for identification of aspects, and traceability of those aspects to (and from) the design. For this reason, our related work primarily describes work on identification of aspects from requirements; we do not focus on standalone aspect-oriented design approaches. Previous publications on Theme/UML $[4,6,5]$ describe other work on design.

\subsection{Aspect-Oriented Requirements Engineering}

There have been several efforts in capturing and relating aspect-oriented requirements $[16,18,8,13,11,10,3]$. Here we consider the two which relate most closely to the Theme approach.

Rashid et al [13] provide the AORE (Aspect-Oriented Requirements Engineering) model and ARCaDe (Aspectual Requirements Composition and Decision support) approach and tool for describing components and requirements-level aspects. Examples of these aspects are compatibility, availability, or security. This work builds on the ViewPoints model [9], which is intended to support the integration of heterogeneous requirements specified from multiple perspectives. An early stage in the AORE model is the identification and specification of concerns. The approach to this differs from the Theme approach to concern identification in that it relies on the domain knowledge of the developer to identify possible non-functional requirements to be taken into account when implementing a particular requirement. Those concerns are not explicitly mentioned in the requirements specification; it is up to the developer to ascertain their relevance on their own. We see this as a complemetary approach to our own. Such domain knowledge will always play a large part in system design. The Theme/Doc approach aims to support the analysis of relationships between behaviours described in requirements specifications. It is possible that the Theme/Doc approach to aspect identification could be used during the concern identification phase of AORE, or could support AORE's extension to include functional as well as non-functional requirements.

Katera and Katz [11] propose architectural views of aspects as a means for reasoning about the relationships among aspects in a system. They describe aspects as crosscutting augmentations to an existing design. In particular, they allow for specification of the overlap between aspects through the concept of a sub-aspect that provides the overlapping functionality, and they make relationships between aspects explicit. A UML approach is given to support these views which differs from the Theme/UML approach: it provides additional architectural support for aspect modelling to that provided by Theme/UML, and it uses aspect mappings rather than multi-dimensional composition style semantics. Theme/Doc could be integrated into this approach since the relationships exposed between behaviours in a set of requirements could be used to establish the behaviours between aspects and sub-aspects in this approach, as well as support the identification of functionality shared between components.

\subsection{Concept Graphing}

The graphical approach employed by Theme/Doc is similar in spirit to conceptual graphs (CGs), as introduced by Sowa [14]. These are visual systems of logic that are readable by humans. CGs provide a formal graphical representation of concepts and the relationships between concepts. CGs have been used for automating consistency checking in multiple-perspective software specifications [15]. This work differs from the approach used in Theme/Doc in that it is concerned with the integration of heterogeneous requirements, whereas we are focused on providing views which expose relationships between functions and entities described in requirements. More investigation would be needed to see whether the formalisms employed by these techniques could be integrated into the Theme/Doc approach as a way to blend it with aspect-oriented requirements engineering approaches.

The Theme/Doc approach draws from experience developing the Design Pattern Rational Graph [2] (DPRG) approach and tool, which provides developers with a way to link high-level concepts described in a design pattern with their implementation in source. Part of a DPRG graphically represents sentences from design pattern text. This representation differs from the views offered in Theme/Doc. These approaches also differ in intent: the pattern 
level graph of a DPRG is intended to assist a developer in understanding an existing design by decomposing its textual description and relating it to design and implementation. Theme/Doc provides visual cues for requirements analysis, and aspect identification, composition, and design. As such, the views presented in this paper are designed to map directly to Theme/UML, and to expose relationships in requirements text to facilitate identification of aspects.

\section{Conclusions}

In order to identify aspects in a set of requirements and map them to design, we need to see how behaviours described in the requirements relate to one another. In this paper we have presented the Theme approach, which provides a model and tool support for identification of aspects in requirements specifications, design level modelling of aspects and their composition, and checking design decisions in the context of the requirements. The Theme approach is based on Theme/UML which is augmented by the Theme/Doc process presented here. Theme/Doc provides four views of a requirements specification: the action view; the clipped action view, which clusters requirements with particular behaviour, and shows which actions were chosen to crosscut others; the theme view, which depicts the entities and actions of a cluster from the clipped view; and the augmented theme view, which places design decisions into the context of the requirements. Our case study showed that this approach is effective in helping to identify aspects in requirements specifications. In addition, these views provide traceability links from the requirements to the design as modeled in Theme/UML. We also found that the approach could be used to check coverage of requirements in design, and we identified functionality that would enhance the scalability of the approach.

\section{Acknowledgements}

We would like to thank Conor Ryan, Alan Gray, David McKitterick, Karl Quinn and Tonya McMorrow from the original Crystal Game development team, on which our case study was based. Also thanks to Mary Lee for early work applying the Theme approach, and to Cormac Driver and Ryan van Roode for comments on earlier drafts of this paper. We would also like to thank the anonymous reviewers for their comments and insight.

\section{References}

[1] Aspectj home page, Xerox PARC, USA, http://aspectj.org/.

[2] E. Baniassad, G. Murphy, and C. Schwanninger. Design pattern rationale graphs: Linking design to source. In Proceedings of the International Conference on Software Engineering, pages 352-362, 2003.
[3] J. Castro, M. Kolp, and J. Mylopoulos. Towards requirements-driven information systems engineering: The tropos project, 2002.

[4] S. Clarke. Extending standard uml with model composition semantics. Science of Computer Programming, 44(1):71100 , July 2002

[5] S. Clarke and R. Walker. Towards a standard design language for AOSD. In Proceedings of the International Conference on Aspect-Oriented Software Development, pages 113-119. ACM Press, 2002.

[6] S. Clarke and R. J. Walker. Composition patterns: An approach to designing reusable aspects. In International Conference on Software Engineering, pages 5-14, 2001.

[7] Y. Coady and G. Kiczales. Back to the future: A retroactive study of aspect evolution in operating system code. In Proceedings of the International Conference on Aspect-oriented Software Development, pages 50-59, 2003.

[8] R. Darimont and A. van Lamsweerde. Formal refinement patterns for goal-driven requirements elaboration. In Foundations of Software Engineering, pages 179-190, 1996.

[9] A. Finkelstein. The viewpoints faq. BCS/IEE Software Engineering Journal, 11(1), 1996.

[10] J. Grundy. Aspect-oriented requirements engineering for component based software systems. In 4th IEEE International Symposium on Requirements Engineering, pages 8491.

[11] M. Katera and S. Katz. Architectural views of aspects. In Proceedings of the International Conference on Aspectoriented Software Development, pages 1-10, 2003.

[12] E. Koutsofios and S. North. Drawing graphs with dot. Murray Hill, NJ.

[13] A. Rashid, A. Moreira, and J. Araujo. Modularisation and composition of aspectual requirements. In Proceedings of the International Conference on Aspect-oriented Software Development, pages 11-20, 2003.

[14] F. Sowa. Conceptual Structures: Information Processing in Mind and Machine. Addison-Wesley, 1998.

[15] T. T. Sunetnanta and A. Finkelstein. Automated consistency checking for multiperspective software applications. In Proceedings of the International Conference on Software Engineering Workshop on Advanced Separation of Concerns, 2001.

[16] S. Sutton and I. Rouvellou. Modeling of software concerns in cosmos. In Proceedings of the International Conference on Aspect-oriented Software Development, pages 127-133, 2002.

[17] P. Tarr, H. Ossher, W. H. Harrison, and S. S. Jr. N degrees of separation: Multi-dimensional separation of concerns. In Proceedings of the International Conference on Software Engineering, pages 107-119. IEEE Computer Society Press, 1999.

[18] X. Wang and Y. Lesperance. Agent-oriented requirements engineering using congolog and i*. In Submitted to AOIS-2001, Bi-Conference Workshop at Agents 2001 and CAiSE'01., 2001. 